\title{
PERSPECTIVE
}

\section{Repurposing the dual orexin receptor antagonist suvorexant for the treatment of opioid use disorder: why sleep on} this any longer?

\author{
Morgan H. James $\mathbb{I D}^{1,2}$, Jennifer E. Fragale ${ }^{1}$, R. Nisha Aurora ${ }^{3}$, Nina A. Cooperman ${ }^{4}$, Daniel D. Langleben ${ }^{5,6}$ and $^{\text {Gary }}$ Aston-Jones ${ }^{1}$ \\ Neuropsychopharmacology (2020) 45:717-719; https://doi.org/10.1038/s41386-020-0619-x
}

In 2017 the US Department of Health and Human Services declared the epidemic of opioid use disorder (OUD) sweeping the nation a public health emergency. Major aspects of OUD management include detoxification, prevention of overdose, and relapse prevention. The main approach to OUD treatment is opioid substitution therapy (OST) using full (methadone) or partial (buprenorphine) opioid agonists. Drawbacks of this approach include restricted access, risk of overdose, stigma of iatrogenic opioid dependence, and interference with pain management that limit its effectiveness. Clearly, there is an urgent need for the development of new medications that rely on different mechanisms, which could augment OST or serve as an alternative. In 2018, the National Institute on Drug Abuse's (NIDA) Division of Therapeutics and Medical Consequences named ten mechanisms of action, which NIDA has gauged to have the "highest probability of a path to FDA approval for the treatment of some aspect of OUD in the near term" [1]. Atop this list are antagonists and/or negative allosteric modulators that dampen the signaling of the hypothalamic orexin (hypocretin) neuropeptides. We believe this focus to be well-justified given the $15+$ years of preclinical data indicating that orexin signaling, particularly at the orexin-1 receptor $(\mathrm{O} \times 1 \mathrm{R})$, selectively underlies the motivational and craving properties of all drugs of abuse tested [2].

We and others have speculated that a rapid orexin-based approach might be to repurpose the already FDA-approved dual Ox1R/Ox2R antagonist, suvorexant, marketed by Merck as Belsomra ${ }^{\mathrm{TM}}$ for the treatment of insomnia [2-4]. In addition to reducing drug craving (primarily via actions at Ox1R), suvorexant may have the additional benefit of indirectly reducing relapse risk by normalizing sleep disturbances (primarily via Ox2R), which are observed in $\sim 80 \%$ of OUD patients. Indeed, the wellknown deficits in executive function that accompany disturbed sleep may contribute to relapse vulnerability in treatmentseeking OUD individuals, as sleep disturbance comorbidity is negatively associated with OST outcomes [5]. Moreover, given the known role for the orexin system in mediating stress and anxiety behaviors [6], including opioid withdrawal syndrome [7], additional therapeutic benefits of suvorexant may come from its ability to treat underlying comorbid disorders. Several clinical trials are currently underway to examine the efficacy of suvorexant in normalizing sleep outcomes, as well as craving, stress, and sleep indices, in OUD and other substance use disorder patients (ClinicalTrials.gov Identifier numbers: NCT03412591, NCT03897062, NCT03789214, NCT03937986, NCT03789214, NCT03657355). These studies represent an important step toward repurposing suvorexant for addiction, and will provide proof-of-principle data to help guide the development of further trials across various substances of abuse. However, as we outline here, preclinical studies examining systemic dual orexin receptor antagonism as a therapeutic for opioid addiction remain nascent, and several key questions are unanswered with respect to the efficacy and safety profile of suvorexant in OUD individuals.

Notably, no clinical or preclinical study has yet directly tested the efficacy of suvorexant in reducing opioid use and craving. Indeed, all three studies that explored suvorexant as a treatment for addiction were conducted in rodent models of psychostimulant abuse [8]. This being said, many animal studies now support the efficacy of selective orexin antagonists in reducing a wide range of OUD-related behaviors, including demand and relapse (e.g., see [9]. However, these studies have generally focused on selective Ox1R antagonists, and thus it is unclear whether suvorexant, which has a slightly higher binding affinity for $\mathrm{O} 2 \mathrm{R}$ $(0.35 \mathrm{nM})$ over Ox1R (0.55 nM) [10], can reduce craving without also causing unwanted sedation or off-target effects. Notably, one study reported that $\mathrm{O} 2 \mathrm{R}$ antagonism reduced heroin intake following long duration drug self-administration sessions [11], indicating that there may be therapeutic benefit to suvorexant's actions at both receptors.

Key questions also remain regarding the prospective dosing regimen of suvorexant, as the overwhelming majority of studies of selective or dual orexin receptor antagonists have tested their efficacy only when administered acutely. This is important, as one study reported that chronic Ox1R antagonism during cocaine extinction training resulted in enhanced cued reinstatement when tested in a drug-free state [12]. Also, new evidence indicates that a single dose of an Ox1R antagonist can disrupt drug seeking behavior beyond the bioavailability of the compound [13], raising the possibility that daily dosing may not be necessary. It is also unclear what time of day would be best to administer suvorexant; when prescribed for insomnia, suvorexant is administered at bedtime; however, the $\sim 12 \mathrm{~h}$ half-life of suvorexant means that this regimen might result in reduced bioavailability during the daytime when opportunity for drug use is high. It is possible that

\footnotetext{
${ }^{1}$ Brain Health Institute, Rutgers University, Piscataway, NJ, USA; ${ }^{2}$ Florey Institute of Neuroscience and Mental Health, University of Melbourne, Parkville, VIC, Australia; ${ }^{3}$ Department of Medicine, Rutgers Robert Wood Johnson Medical School, New Brunswick, NJ, USA; ${ }^{4}$ Division of Addiction Psychiatry, Rutgers Robert Wood Johnson Medical School, New Brunswick, NJ, USA; ${ }^{5}$ Perelman School of Medicine, University of Pennsylvania, Philadelphia, PA, USA and ${ }^{6}$ Philadelphia VA Medical Center, Philadelphia, PA, USA Correspondence: Gary Aston-Jones (aston.jones@rutgers.edu)
}

Received: 25 October 2019 Revised: 9 December 2019 Accepted: 30 December 2019

Published online: 27 January 2020 
twice a day dosing of suvorexant might counteract this problem; however, lower doses might be necessary to avoid impairments in daytime wakefulness. Relatedly, in a study of healthy adults, a subgroup of patients reported daytime somnolence at $9 \mathrm{~h}$ postdosing which was associated with impaired driving ability [14]. Thus, patients are advised against next-day driving and other activities requiring full alertness following $20 \mathrm{mg}$ suvorexant, raising potential issues associated with compliance. Fortunately, recent preclinical results indicate that low doses may be effective for reducing drug craving, as Ox1R antagonists are more effective at lower doses in rats that exhibit a strong addiction phenotype $[9,15]$. This would need to be tested explicitly for suvorexant. It is also possible that suvorexant could be used as an adjunct to existing OUD medications, however, significant preclinical work is required to identify the optimal dosing regimen to maximize efficacy and minimize side effects. An additional possible clinical application includes the coadministration of suvorexant with opioid-based pain medications to prevent drug-induced plasticity in reward regions that might contribute to the development of addiction [16], although this possibility remains to be tested in animal models.

Prescribing suvorexant in OUD populations also raises safety concerns that are yet to be addressed by animal studies. One important concern is the potential for suvorexant, whether alone or in combination with conventional opioid substitution therapies, to exacerbate or precipitate sleep-disordered breathing and other respiratory complications that are commonly observed in OUD patients. Chronic opioid use is associated with a direct reduction in ventilation, due in part to $\mu$ receptor activation in pre-Botzinger nucleus, the presumed site of respiratory rhythm genesis [17]. Orexin neurons directly innervate central autonomic and respiratory regions, including pre-Botzinger complex as well as phrenic motoneurons [18]. Orexin knockout mice exhibit compromised chemoreflexes in response to hypercapnic conditions and a higher number of spontaneous apneas during both NREM and REM sleep, and these effects are largely recapitulated in wildtype mice with systemic administration of an Ox1R antagonist [18]. Although orexin-associated respiratory effects have not been fully elucidated in humans, there is an increased prevalence of sleepdisordered breathing in patients with narcolepsy, a sleep-wake disorder associated with loss of orexin neurons [19]. In contrast, suvorexant was reported to have no clinically significant effects in persons with mild-to-moderate obstructive sleep apnea [20]. It is also noteworthy that fewer respiratory effects might be predicted with suvorexant compared with traditional hypnotic agents such as benzodiazepines, which globally activate the GABAergic system in the brain. Regardless, there is a clear need to study the impact of suvorexant on metrics of sleep-disordered breathing such as hypoxemia and the apnea-hypopnea index in persons with OUD, or in those receiving opioid maintenance therapy.

In addition to potential respiratory complications, the hypnotic effects of suvorexant could exacerbate opioid-induced daytime drowsiness, a particular concern if suvorexant is administered during the day to maximize its anti-craving properties. As described above, the low doses needed for effectiveness of OxR1 antagonists in strongly 'addicted' rats $[9,15]$ decrease such concerns. A combination of suvorexant (at night) and a selective OxR1 antagonist (in daytime, with limited sedating properties and therefore potentially fewer contraindications) might be clinically most acceptable.

Finally, it is worth noting that suvorexant is designated a DEA schedule IV-controlled substance in the United States because it is considered to have abuse liability similar to other sleep medications (e.g., zolpidem; also schedule IV). Studies in opioidnaive animals have failed to observe any evidence of withdrawal or "discontinuation syndrome" following abrupt discontinuation of suvorexant, and there is no evidence of suvorexant having positive reinforcing effects in self-administration studies [21], however it is important that these studies are repeated in opioiddependent animals.

In sum, the opioid crisis demands urgent and concentrated efforts to examine the potential benefits of suvorexant for the treatment of opioid use disorder. These efforts should occur at both the preclinical and clinical levels-and preferably in parallel - to address the key outstanding safety and efficacy questions outlined here. Optimism might be drawn from the fact that a recent preliminary, proof-of-concept investigation of suvorexant administration in patients with cocaine use disorder reported some improvement in behavioral domains related to relapse with no major adverse outcomes [22]. This study was largely guided by a solid foundation of preclinical work pointing to a high likelihood of efficacy for cocaine addiction. It should now be a priority to facilitate similar translational studies for opioid addiction.

\section{FUNDING AND DISCLOSURES}

$\mathrm{MHJ}$ is supported by National Institute on Drug Abuse (NIDA; K99DA045765). JEF is supported by the National Institutes of Health (NIH) IRACDA postdoctoral fellowship (K12 GM093854). RNA is supported by the National Heart, Lung and Blood Institute (HL118414). NAC is supported by NCCIH and NIDA (R33 AT010109) and a Laura and Arnold Foundation Grant. DDL is supported by NIDA (R21 DA043983). GAJ is supported by NIDA (R01 DA006214). RNA is on the Board of Directors for the American Academy of Sleep Medicine, The American Academy of Sleep MedicineFoundation, and the American Board of Sleep Medicine. DDL served on the Scientific Advisory Board of Alkermes Inc and US WorldMeds. GAJ has been a consultant for Merck Pharmaceuticals. All other authors have no disclosures to report.

\section{ADDITIONAL INFORMATION}

Publisher's note Springer Nature remains neutral with regard to jurisdictional claims in published maps and institutional affiliations.

\section{REFERENCES}

1. Rasmussen K, White DA, Acri JB. NIDA's medication development priorities in response to the opioid crisis: ten most wanted. Neuropsychopharmacology. 2018. https://doi.org/10.1038/s41386-018-0292-5.

2. James MH, Mahler SV, Moorman DE, Aston-Jones G. A decade of orexin/hypocretin and addiction: where are we now? Curr Top Behav Neurosci. 2017;33:247-81.

3. Campbell EJ, Marchant NJ, Lawrence AJ. A sleeping giant: suvorexant for the treatment of alcohol use disorder? Brain Res. 2018. https://doi.org/10.1016/j. brainres.2018.08.005.

4. James MH, Aston-Jones G. Introduction to the Special Issue: "Orexin/hypocretin receptor antagonists for the treatment of addiction and related psychiatric disease: What are the steps from here?". Brain Res. 2020:146665.

5. Hartwell EE, Pfeifer JG, McCauley JL, Moran-Santa Maria M, Back SE. Sleep disturbances and pain among individuals with prescription opioid dependence. Addict Behav. 2014;39:1537-42.

6. James MH, Campbell EJ, Dayas CV. Role of the orexin/hypocretin system in stressrelated psychiatric disorders. Curr Top Behav Neurosci. 2017;33:197-219.

7. Georgescu D, Zachariou V, Barrot M, Mieda M, Willie JT, Eisch AJ, et al. Involvement of the lateral hypothalamic peptide orexin in morphine dependence and withdrawal. J Neurosci. 2003;23:3106-11.

8. Simmons SJ, Gentile TA. Cocaine abuse and midbrain circuits: functional anatomy of hypocretin/orexin transmission and therapeutic prospect. Brain Res. 2019. https://doi.org/10.1016/j.brainres.2019.02.026.

9. Fragale JE, Pantazis $\mathrm{CB}$, James $\mathrm{MH}$, Aston-Jones $\mathrm{G}$. The role of orexin-1 receptor signaling in demand for the opioid fentanyl. Neuropsychopharmacology. 2019;44:1690-7.

10. Cox CD, Breslin MJ, Whitman DB, Schreier JD, McGaughey GB, Bogusky MJ. et al. Discovery of the dual orexin receptor antagonist [(7R)-4-(5-chloro-1,3-benzoxazol-2-yl)-7-methyl-1,4-diazepan-1-yl] [5-methyl-2-(2H-1,2,3-triazol-2-yl)phenyl] 
Repurposing the dual orexin receptor antagonist suvorexant for the.. $\mathrm{MH}$ James et al.

methanone (MK-4305) for the treatment of insomnia. J Med Chem. 2010;53:5320-32.

11. Schmeichel BE, Barbier E, Misra KK, Contet C, Schlosburg JE, Grigoriadis D, et al. Hypocretin receptor 2 antagonism dose-dependently reduces escalated heroin self-administration in rats. Neuropsychopharmacology. 2015;40:1123-9.

12. Zhou L, Smith RJ, Do PH, Aston-Jones G, See RE. Repeated orexin 1 receptor antagonism effects on cocaine seeking in rats. Neuropharmacology. 2012;63:1201-7.

13. Mohammadkhani A, James MH, Aston-Jones G. Persistent effects of the orexin-1 receptor antagonist SB-334867 on motivation for the fast acting opioid remifentanil. Brain Res. 2019:146461. https://doi.org/10.1016/j.brainres.2019.146461.

14. Vermeeren $A$, Sun $H$, Vuurman EF, Jongen $S$, Van Leeuwen $C J$, Van Oers $A C$, et al. On-the-road driving performance the morning after bedtime use of suvorexant 20 and 40 mg: a study in non-elderly healthy volunteers. Sleep. 2015;38:1803-13.

15. James $\mathrm{MH}$, Bowrey HE, Stopper CM, Aston-Jones G. Demand elasticity predicts addiction endophenotypes and the therapeutic efficacy of an orexin/hypocretin1 receptor antagonist in rats. Eur J Neurosci. 2018. https://doi.org/10.1111/ ejn.14166.
16. Baimel C, Borgland SL. Orexin signaling in the VTA gates morphine-induced synaptic plasticity. J Neurosci. 2015;35:7295-303.

17. Smith JC, Ellenberger HH, Ballanyi K, Richter DW, Feldman JL. Pre-Botzinger complex: a brainstem region that may generate respiratory rhythm in mammals. Science. 1991;254:726-9.

18. Carrive $\mathrm{P}$, Kuwaki T. Orexin and central modulation of cardiovascular and respiratory function. Curr Top Behav Neurosci. 2017;33:157-96.

19. Nishino S, Ripley B, Overeem S, Lammers GJ, Mignot E. Hypocretin (orexin) deficiency in human narcolepsy. Lancet. 2000;355:39-40.

20. Sun H, Palcza J, Card D, Gipson A, Rosenberg R, Kryger M, et al. Effects of suvorexant, an orexin receptor antagonist, on respiration during sleep in patients with obstructive sleep apnea. J Clin Sleep Med. 2016;12:9-17.

21. Born S, Gauvin DV, Mukherjee S, Briscoe R. Preclinical assessment of the abuse potential of the orexin receptor antagonist, suvorexant. Regul Toxicol Pharmacol. 2017;86:181-92.

22. Suchting R, Yoon JH, Miguel GGS, Green CE, Weaver MF, Vincent JN, et al. Preliminary examination of the orexin system on relapse-related factors in cocaine use disorder. Brain Res. 2019. https://doi.org/10.1016/j.brainres.2019.146359. 